\title{
Presence of Connecting Channels in the Western Scheldt Estuary
}

\author{
Cilia M. Swinkels ${ }^{\dagger}$, Claire M.C.J.L. Jeuken ${ }^{\dagger}$ Zheng B. Wang ${ }^{\dagger \dagger}$, and Robert J. Nicholls ${ }^{\S}$ \\ ${ }^{\dagger}$ Deltares/Delft Hydraulics \\ Marine and Coastal Systems/Hydraulic \\ Engineering \\ P.O. Box 177 \\ $2600 \mathrm{MH}$ Delft \\ †TU Delft \\ Faculty of Civil Engineering \\ Stevinweg 1 \\ 2628 CN Delft \\ The Netherlands \\ \$University of Southampton \\ School of Civil Engineering and the \\ Environment \\ Southampton SO17 1BJ \\ United Kingdom
}

The Netherlands

cilia.swinkels@deltares.nl

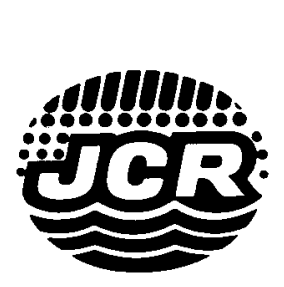

\begin{abstract}
SWINKELS, C.M.; JEUKEN, C.M.C.J.L.; WANG, Z.B., and NICHOLLS R.J., 2009. Presence of connecting channels in the Western Scheldt Estuary. Journal of Coastal Research, 25(3), 627-640. West Palm Beach (Florida), ISSN 07490208 .

To predict the effects of dredging operations in the Western Scheldt estuary, insight into the morphological behaviour of its channels is needed. The estuary features two large main channels that meander alongside each other and that are linked by smaller connecting channels. These connecting channels originate from water level differences between the two main channels. Three hydrodynamic mechanisms are investigated that are responsible for generating such water level differences: (1) differences in tidal wave propagation along two neighbouring main channels, (2) centrifugal forces, and (3) Coriolis forces.

The magnitude of the three mechanisms is analytically assessed based on channel geometry, which is derived from historical depth charts. The analysis demonstrates a large temporal variability of the water level differences due to tidal wave propagation, mainly as a function of changes in the depth ratio between the two main channels. Conversely, both the centrifugal effect and the Coriolis effect are relatively constant over time. The temporal evolution of the connecting channels can therefore primarily be attributed to the wave propagation mechanism. A correlation analysis reveals a linear relationship between connecting channel dimensions and the net water level differences produced by the three processes.

The relationship suggests that dredging operations may significantly influence the evolution of connecting channels by changing the depth ratio between the two main channels. The proposed future deepening of the navigation channel is therefore expected to induce a decline in the size, or even a total disappearance, of connecting channels in some parts of the estuary.
\end{abstract}

ADDITIONAL INDEX WORDS: Morphological evolution, dredging, estuarine management.

\section{INTRODUCTION}

The Western Scheldt is a large coastal estuary situated in the southwest of the Netherlands (Figure 1). Its unique flora and fauna are of large ecological value within Europe. At the same time, the estuary is of vital importance to the Dutch and Belgian economy by offering navigation routes to the ports of Gent, Terneuzen, Vlissingen, and especially Antwerp. The frequently conflicting economic and environmental interests make the management of the estuary a complex task. Collaboration between the Belgian and Dutch governments has resulted in the formulation of a Long-Term Vision (LTV) for the Western Scheldt. The primary management objective for the estuary described in the LTV is "to preserve the physical characteristics and dynamics of the Western Scheldt channel system."

The morphology of the Western Scheldt is characterized by an ebb-flood channel system, which was described by Van Veen (1950) as a braided channel pattern (Figure 2). He

DOI: 10.2112/06-0719.1 received 20 June 2006; accepted in revision 18 April 2008. sketches the system as a mutually evasive ebb and flood channel that form a regular meandering pattern, which is often referred to as a "multichannel system." In Van Veen's definition, the flood channel is open to the flood current and exhibits a sill at the upstream end. Conversely, the ebb channel is open to the ebb current and exhibits a sill at the seaward end. In most parts of the estuary, the curved ebb channel is deeper than the flood channel and provides the main navigational route to the port of Antwerp. The flood channel is relatively straight and forms a shortcut through the inner bend of the meandering ebb channel. Where the flood and ebb channels meet, a shallow bar area is present.

The multichannel system can be schematized as a series of repetitive units, each of which comprises a curved ebb channel section and a straight flood channel section, separated by an intertidal or subtidal shoal. These units can be regarded as distinct morphological cells and can be defined as such on the basis of the residual sediment circulation, which results from the asymmetric water motion through the ebb and flood channel (Jeuken, 2000; Winterwerp et al., 2001). Six of such "macrocells" can be distinguished in the Western Scheldt 


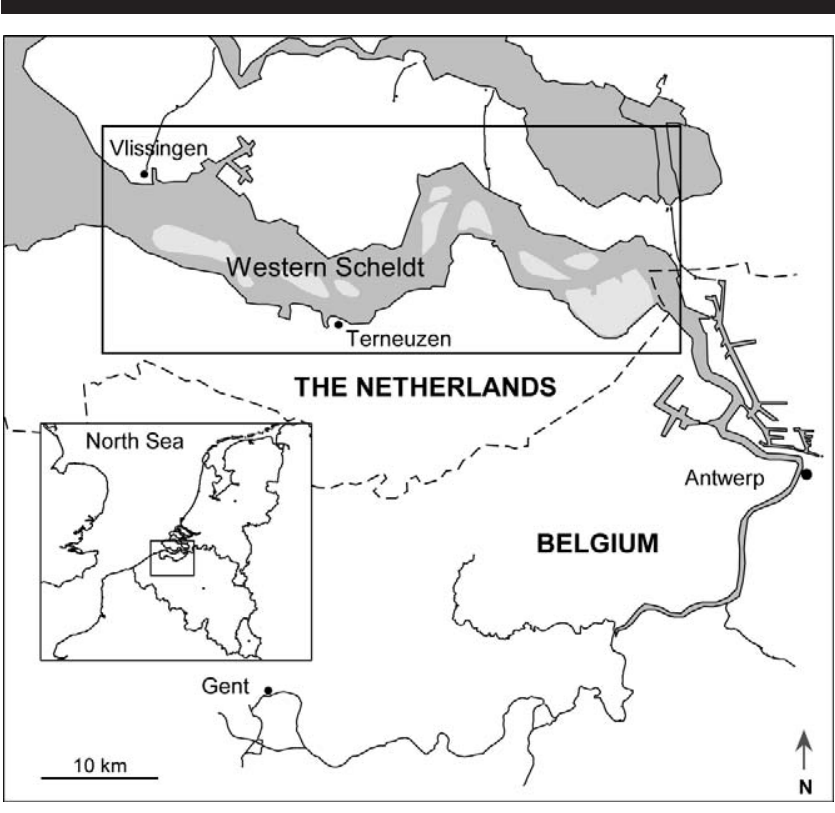

Figure 1. The Western Scheldt study area.

(Figure 3), which are successively labelled macrocell 1 to 6 from west to east.

Connecting channels provide a link between a main ebb and flood channel by intersecting the shallow sub- and intertidal areas between the two channels and are unique morphological features of the multichannel system. They induce water exchange between two main channels and thereby redistribute the tidal flow in the channel system (Jeuken, 2000). Water level differences between a main ebb and flood channel provide the driving force of the flow in the connecting channel and are hence responsible for maintaining the connecting channels (Van den Berg, Jeuken, and Van der Spek, 1996). Connecting channels are present in areas where water level differences are largest, which is typically the bar area at the landward end of the flood channel (Jeuken, 2000). Connecting channels cutting through this bar area are referred to as bar connecting channels. Additionally, a second type of connecting channels can be distinguished in the Western Scheldt: the shoal connecting channels, which cross through the shallow intertidal area separating the two main channels (Figure 3). Larger water level differences are likely to be associated with larger and/or more connecting channels. Connecting channels tend to display a quasi-cyclic behaviour, characterized by stages of expansion, migration, and degeneration. They are marked by rapid lateral migration (with rates up to $100 \mathrm{~m} / \mathrm{y}$ ), which makes them the most dynamic elements in the channel system.

During the past century, maintenance of the navigation channel, dumping of dredge spoil, and sand extraction have had a profound influence on the morphology of the Western Scheldt. Because of these operations, shallow water areas have been lost, the main channels have generally become deeper, and the smaller connecting channels have diminished in size due to sedimentation and lost (part of) their mobility.

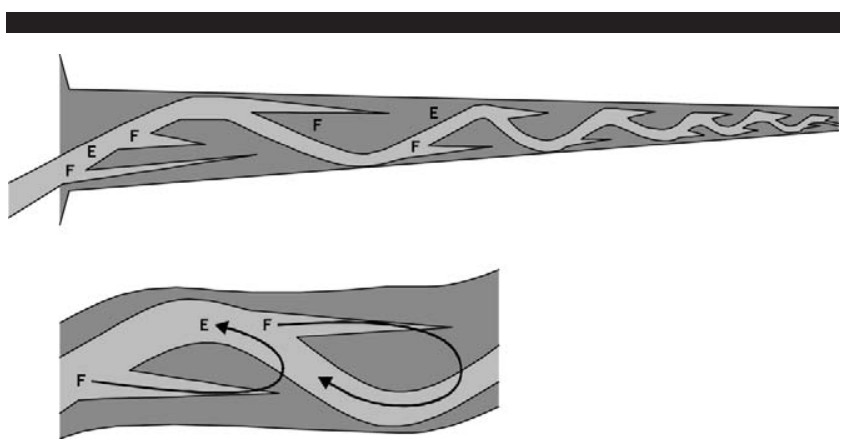

Figure 2. Van Veen's (1950) braided channel pattern (top) and the residual sediment circulation over the main channels (bottom). $\mathrm{F}=$ flood channel, $\mathrm{E}=$ ebb channel.

From the LTV perspective, this is an undesirable development. Nonetheless, a new deepening of the navigation channel has been negotiated between the Dutch and Belgian authorities, which will again involve major capital dredging works. As further deterioration of the multichannel system should be prevented, the future dredging scheme needs a well-considered strategy. To predict the effects of the dredging operations and to evaluate the current state of the Western Scheldt, it is proposed to monitor physical indicators that are characteristic of the state of the multichannel system. Insight into the response of an indicator to (human) alterations of the system would facilitate more informed decisionmaking in management issues (Van Koningsveld, Davidson, and Huntley, 2005). One of the proposed indicators to monitor the Western Scheldt is the presence of the connecting channels (Jeuken and Wang, 2009; Wang, Jeuken, and Winterwerp, 2009) as their presence and dynamic behaviour are considered favourable for the estuary; they provide routes for inland shipping traffic, which improves the navigability of the estuary, and their migration is considered to revitalize shallow intertidal/subtidal areas, which is beneficial for the ecology.

Until now, the only research work that explicitly addressed connecting channels was that by Jeuken (2000), describing the morphodynamic behaviour of the connecting channels in the Terneuzen section (macrocell 2). The aim of this article is to provide more insight into the general factors controlling connecting channels and their response to dredging within the estuary. Whereas the morphological development of the main channels is reasonably well understood (e.g., Wang et al., 2005; Winterwerp et al., 2001), the behaviour of the connecting channels is more difficult to predict, as it is governed by a system of complex feedback mechanisms forced by the hydrodynamic processes in the main channel. In this study, it is attempted to relate the development of the meso-scale connecting channels to changes in the macroscale main channels. This is achieved by an analysis of the hydrodynamic mechanisms that maintain connecting channels and the influence that the main channels exert on these phenomena. This article presents the results of the investigation and discusses the relationship between main and connecting channels in light of the dredging operations in the estuary. 


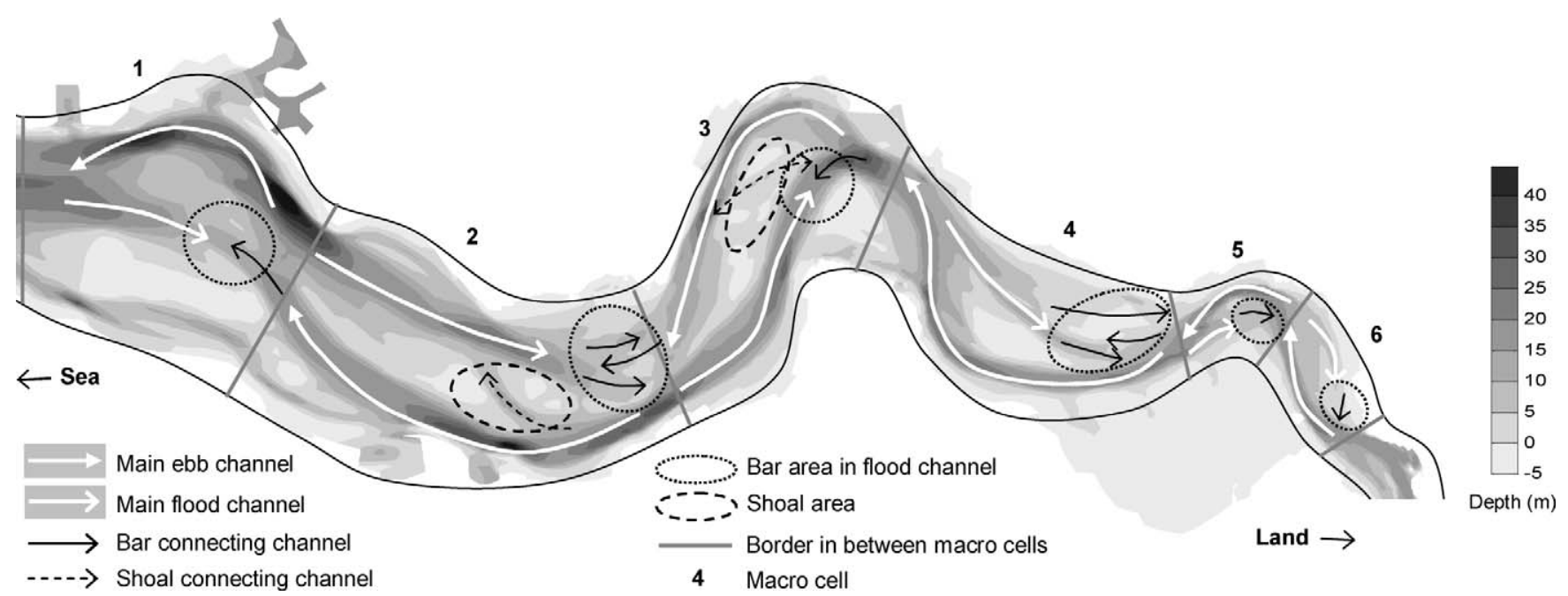

Figure 3. Channel types and bar areas in the six macrocells in the Western Scheldt, bathymetry 2002 .

\section{STUDY AREA}

\section{General Description}

The Western Scheldt is a tide-dominated, well-mixed estuary located in the Delta region of the Dutch coast and forms the marine end of the river Scheldt (Figure 1). It covers an area of about $300 \mathrm{~km}^{2}$. The funnel-shaped estuary decreases exponentially in cross-sectional area, and its width reduces from $6 \mathrm{~km}$ at the mouth to about $100 \mathrm{~m}$ near the tidal limit at the sluices of Gent, some $160 \mathrm{~km}$ further upstream. The water motion in the estuary is forced by a semidiurnal progressive tide in the North Sea, which travels from south to north along the Dutch coast. The tidal wave is amplified as it travels up the estuary; the mean tidal range increases from $3.8 \mathrm{~m}$ at Vlissingen to $5.2 \mathrm{~m}$ at Antwerp, $78 \mathrm{~km}$ upstream. The distortion of the tidal wave as it travels landwards is accompanied by a phase shift between the horizontal and vertical tide of about 2.5 to 3 hours. The mean river outflow is approximately $120 \mathrm{~m}^{3} / \mathrm{s}$, which is less than $1 \%$ of the tidal prism (Wang et al., 2002).

\section{Dredging and Dumping Operations}

Since 1920, dredging operations have been carried out in the main ebb channel to provide an access route to the various ports along the estuary. The dredging activities were always most intense in the eastern part of the estuary, where shallow sills in the ebb channel form the main barriers to navigation. The dredged material was generally disposed elsewhere in the estuary, in order not to remove the sediment from the system permanently. Typically, the nearby shallower flood channels and connecting channels served as dumping locations. When this practice appeared to have a large impact on the stability of the channels in the eastern macrocells, dredged sediments were dumped further away in the western part of the estuary.

In addition to maintenance dredging, two large-scale deep- ening programmes were implemented as a response to the increase in vessel draft and traffic density in the estuary. During the first deepening (1970-1975), the navigation channel was deepened to secure a minimal tidal-free depth of the sills of $9.5 \mathrm{~m}$. The second deepening programme was carried out in the period 1997-1998 and increased the tidal-free water depth to $11.6 \mathrm{~m}$. Recently, a new deepening of the navigation channel has been agreed, which will again lower the shallow areas in the navigation channel by approximately 1.5 $\mathrm{m}$ to a tidal-free water depth of $13.1 \mathrm{~m}$. It is estimated that an additional 14 million $\mathrm{m}^{3}$ of sand will be dredged and redeposited throughout the estuary in this upcoming operation.

\section{METHODOLOGY}

\section{Introduction}

As discussed above, connecting channels are maintained by water level differences between the main ebb and flood channels. Van den Berg, Jeuken, and Van der Spek (1996) propose four hydrodynamic mechanisms that may generate these water level differences (Figure 4): (1) differences in channel geometry between a main ebb and flood channel, resulting in differences in tidal wave propagation along them; (2) centrifugal forces; (3) Coriolis forces; and (4) loss of momentum.

In order to establish a morphological relationship between the main channels and connecting channels, these four mechanisms were quantified based on a morphological analysis of the main channels. To this end, the schematization of Winterwerp et al. (2001) of the Western Scheldt, dividing the estuary into six macrocells, was adopted. The macrocells were further subdivided into a main ebb channel, a main flood channel, and connecting channels. The full analysis was based on a bathymetrical data set of the Western Scheldt with yearly surveys dating from 1955 to 2002 .

For each year and each macrocell, various geometrical parameters describing the main channels were derived from the bathymetrical charts, which were then used for quantifica- 


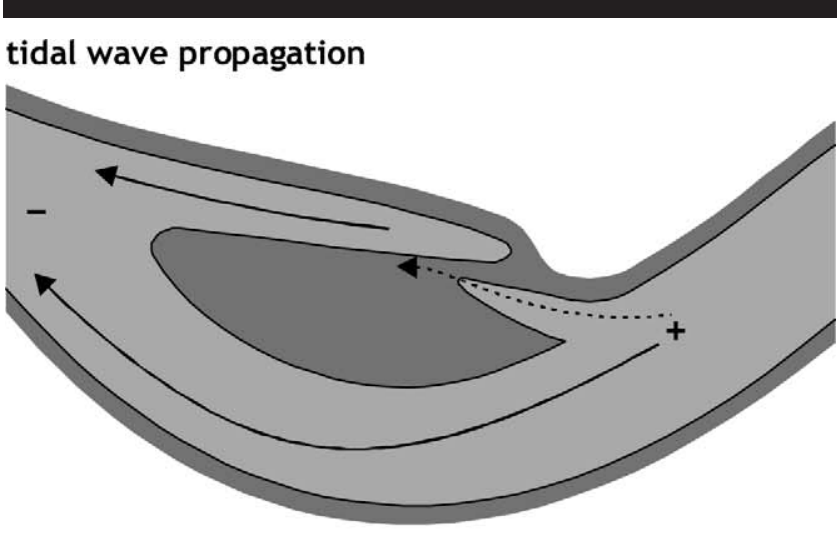

coriolis force
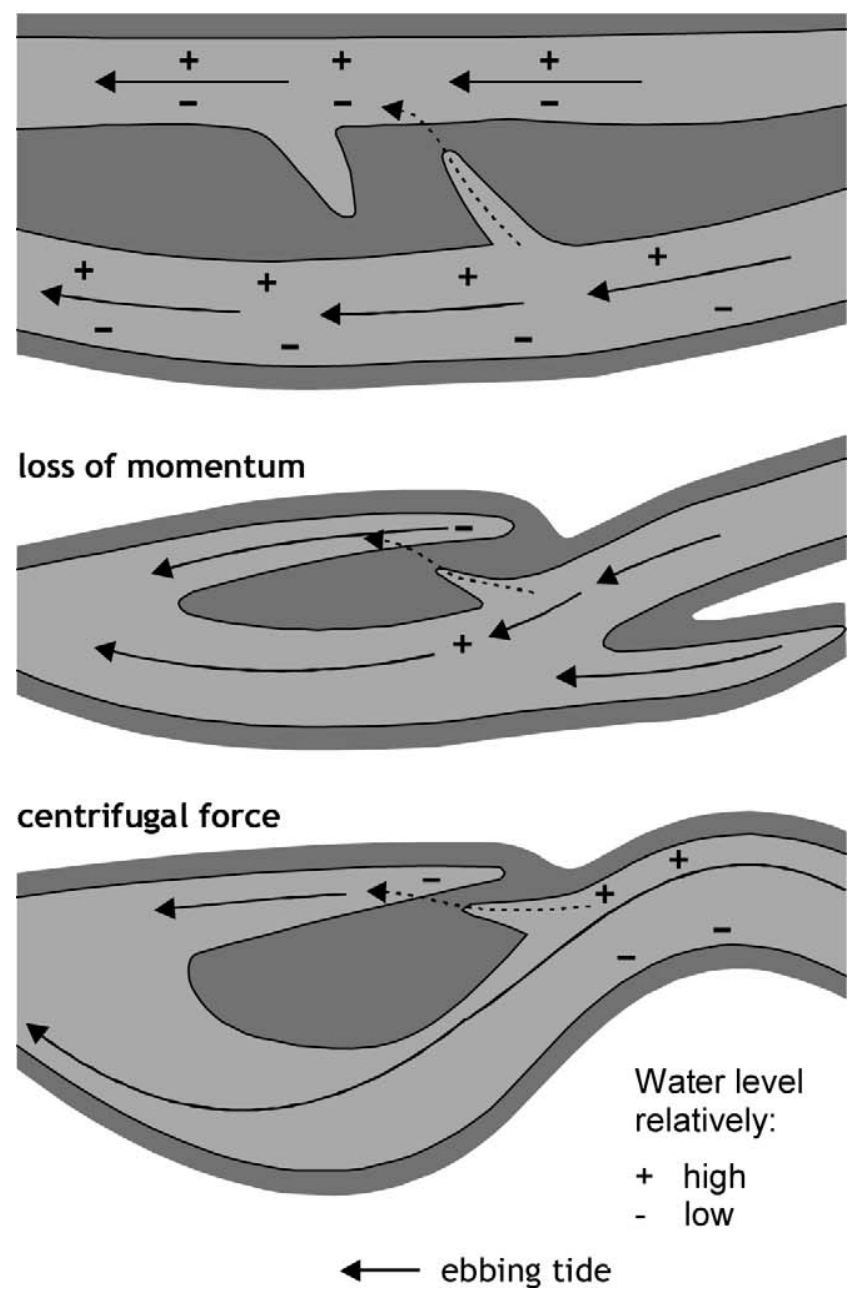

Figure 4. Mechanisms causing water level difference between main channels and the occurrence of connecting channels (after Van den Berg, Jeuken, and Van der Spek, 1996).

tion of the above mechanisms. Next, the connecting channels were identified from the bathymetrical charts and quantified by their cross-sectional area. Subsequently, the correlation between the analytically determined water level differences and size of the connecting channel was tested over the analysis period (1955-2002). Finally, to gain confidence in the analytical assessment of the water level differences, the analytical approach was verified against a hydrodynamic simulation of the Western Scheldt, performed with the numerical model Delft3D-FLOW. A more detailed description of the study approach is given in the following sections.

\section{Mechanisms Causing Water Level Differences}

Of the four hydrodynamic mechanisms, it was hypothesized that the first mechanism, tidal wave propagation, would play a dominant role in the evolution of connecting channels because of its anticipated large temporal variability. From now, it will therefore be referred to as the "primary mechanism." Both the second and third mechanisms induce cross-channel water level differences in the main channels. Jointly, they will be referred to as the "secondary mechanisms" because of their presupposed more stable nature. The fourth mechanism, loss of momentum, was neglected in this study: the water level differences due to this mechanism are approximately an order of magnitude smaller than differences due to the other mechanisms, as in estuaries the Froude number is generally low. The contribution of the three other mechanisms in generating water level differences can be estimated as follows.

\section{Tidal Wave Propagation}

In shallow water, the propagation velocity of the tidal wave $C$ can be approximated by:

$$
C(h)=\sqrt{\frac{g A_{s}(h)}{B(h)}},
$$

where $g$ is acceleration due to gravity $\left(\mathrm{m} / \mathrm{s}^{2}\right), A_{\mathrm{s}}$ is current conveying cross section $\left(\mathrm{m}^{2}\right)$, and $B$ is channel width $(\mathrm{m})$. The factor $A_{\mathrm{s}} / B$ denotes the average channel depth, $h_{a v}$, which varies with tidal level and depends on the form of the channel's hypsometric curve.

Generally, the main ebb and flood channels in a macrocell have a different channel depth $\left(h_{a v}\right)$ and channel length $(L)$. This results in a different propagation time of the tidal wave through the channels and hence causes a water level difference between them. The magnitude of the water level difference due to wave propagation $\left(\Delta h_{w p}\right)$ between two channels can be approximated by:

$$
\Delta h_{w p}=a \Delta t \frac{2 \pi}{T}
$$

where $a$ is tidal amplitude (m), $T$ is tidal period (s), and $\Delta t$ is the difference between the propagation time of the tidal wave in the ebb channel (ec) and the flood channel (fc), given by:

$$
\Delta t=L_{e c} / C_{e c}-L_{f c} / C_{f c} .
$$

Here, $L$ is channel length (m). $\Delta t$, and hence $\Delta h_{w p}$, is defined in such a way that a positive value indicates faster wave propagation along the flood channel, accompanied by a higher water level in the flood channel during rising tide and vice versa during falling tide. 
Annual values of $\Delta h_{w p}$ from 1955 to 2002 were calculated for each macrocell by considering the main channel geometrical variables $L$ and $h_{a v}$, derived from the bathymetrical charts, and annual tidal data dating back to 1955 .

\section{Centrifugal Force}

Centrifugal forces in a channel bend induce a transverse water surface slope. From the equations of continuity and motion for fully developed flow in the middle part of a wide rectangular bend, the transverse water surface slope can be approximated by (e.g., Jansen, 1979):

$$
i_{r}=\left(1+3 \alpha^{2}-2 \alpha^{3}\right) \frac{\bar{u}^{2}}{g R},
$$

where $\bar{u}$ is the cross-channel averaged current velocity, and $R$ is the radius of curvature. The coefficient $\alpha$ is defined by $\sqrt{g} / \kappa C$. Here, $C$ is the Chézy coefficient, and к (Von Karman's constant) is 0.4 . For $C=50 \mathrm{~m}^{0.5} / \mathrm{s}$, this results in a transverse water level difference due to centrifugal forces $\left(\Delta h_{c f}\right)$ over the channel width $(B)$ of:

$$
\Delta h_{c f}=1.06 \frac{\bar{u}^{2}}{g R} B
$$

\section{Coriolis Force}

The transverse water level difference caused by the Coriolis effect $\left(\Delta h_{c o r}\right)$ can be approximated by (e.g., Van Rijn, 1990):

$$
\Delta h_{c o r}=\frac{f \bar{u}}{g} B,
$$

where $f=2 \omega \sin (\varphi)$ is the Coriolis coefficient, $\omega$ is radian frequency, $\phi$ is latitude, and $\bar{u}$ is the cross-channel averaged current velocity.

To assess the magnitude of $\Delta h_{c f}$ and $\Delta h_{c o r}$, the geometrical channel characteristics $R$ and $B$ were derived from the bathymetrical data set for each year. Both variables were assessed for each macrocell at $0 \mathrm{~m}$ NAP (Dutch Ordnance Datum). Additionally, to evaluate the two mechanisms, cross-channel averaged current velocities $(u)$ were also required. As information on temporal or spatial variation of the current velocity throughout the estuary was not available for this study, a constant current velocity of $1 \mathrm{~m} / \mathrm{s}$ was assumed.

\section{Combined Effect of Centrifugal and Coriolis Forces}

Both the centrifugal and Coriolis forces generate a transverse water slope across a channel. The two forces oppose or combine depending on the configuration of the main channels and the direction of the tidal flow and are jointly responsible for a single cross-channel water level difference: the secondary effect. The local secondary effect within the individual channels may generate an overall water level difference over the macrocell (Figure 5), which can be split into a longitudinal $\left(\Delta h_{\text {long }}\right)$ and transverse $\left(\Delta h_{\text {cross }}\right)$ component.

The overall longitudinal component $\Delta h_{\text {long }}$ arises from secondary water level differences at the start and/or end of a channel, responsible for setting up a longitudinal head over

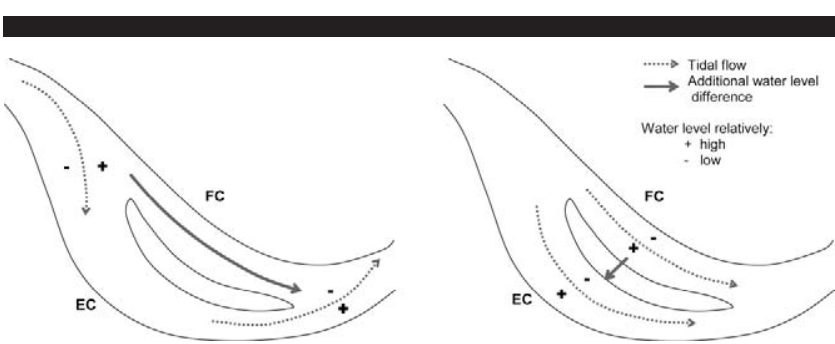

Figure 5. Secondary mechanisms split in a longitudinal effect $\left(\Delta h_{\text {long }}\right.$ (left), and transverse effect $\left(\Delta h_{\text {cross }}\right.$ (right). $+=$ relatively high water level, - = relatively low water level, $\mathrm{EC}=$ ebb channel, $\mathrm{FC}=$ flood channel.

the inside of an estuarine bend. This effect typically reduces the head difference over the ebb channel and increases the head difference over the flood channel during flooding conditions. During falling tide, the effect is less pronounced or may even be opposite because of the horizontal configuration of the channels and the reversal in flow conditions. The magnitude of the resultant $\Delta h_{\text {long }}$ can be deduced from the secondary mechanisms that operate locally at the bifurcation and confluence of an ebb and flood channel and depends strongly on the configuration of the main channels and estuarine bends in the macrocells.

The overall transverse component $\Delta h_{\text {cross }}$ arises from crosschannel water level differences in the main channels on either side of a shoal area. This results in the generation of a head difference over the shoal area, typically with a higher water level on the flood channel side. The magnitude of $\Delta h_{\text {cross }}$ is determined by the local secondary mechanisms operating in the two parallel main channels.

\section{Quantification of Connecting Channels}

To quantify the cross-sectional area of the connecting channels in each macrocell, first of all a manner to identify their boundaries had to be formulated. For this, use was made of the migrational behaviour of the connecting channels, which contrasts strongly with the static position of the main channels (Figure 6), a result of the difference in spatial and temporal evolution scales between the two channel types. By plotting the historical positions of a characteristic depth line, in this case the $-5 \mathrm{~m}$ depth contour, an area with large channel migration can be distinguished in each macrocell. Channels falling within this area were subsequently categorized as connecting channel. Experimenting with different contour levels for the definition of the connecting channel areas proved not to influence the results significantly.

Following this approach, all macrocells exhibit connecting channels in the bar area towards the end of the flood channel, referred to as bar connecting channels. Additionally, macrocells 2 and 3 exhibit a connecting channel in the shoal area separating the two main channels, referred to as shoal connecting channels. The two types of connecting channels are treated independently in the course of the analysis. Figure 3 indicates all areas in the Western Scheldt that contain connecting channels.

Next, the characteristic dimensions of the connecting chan- 


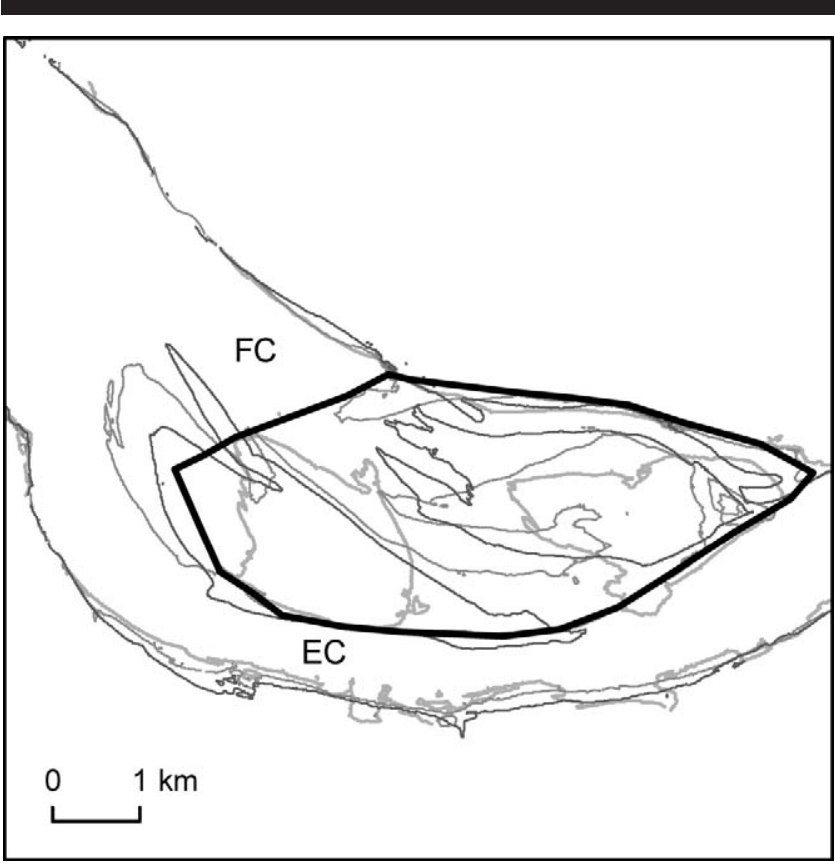

Figure 6. Definition of connecting channel area based on investigation of the migration of $-2 \mathrm{~m}$ depth contours, example of macrocell 4 . From light to dark: contours 1955, 1980, 2000. The black line indicates the resulting bar connecting channel area.

nels, volume and length, were obtained from the charts for each year from 1955 to 2002. These characteristics were used to quantify the connecting channels by their spatially averaged cross-sectional area $(A)$, referenced to NAP $-2 \mathrm{~m}$ :

$$
A=V_{N A P-2 m} / L_{a v} .
$$

where $V$ is total channel volume referenced to NAP $-2 \mathrm{~m}$ $\left(\mathrm{m}^{3}\right)$, and $L$ is length $(\mathrm{m})$. The $-2 \mathrm{~m}$ depth was chosen here as this proved to be an appropriate depth to demarcate between channels and shoals. This is supported by an inflection point in the hypsometric curves of the macrocells showing a rapid increase in surface area above the $-2 \mathrm{~m}$ depth, indicating the presence of shoals above this depth.

\section{Correlation Between Water Level Differences and Connecting Channels}

To explore the relationship between connecting channels and main channels, both the water level difference mechanisms and connecting channels were described by a single representative annual value. In the abovementioned analytical treatment of the mechanisms, therefore, a representative tidal condition for the year was analysed only. This tidal cycle was selected based on a $10 \%$ increase of the mean annual tidal amplitude, which showed to be representative for the Western Scheldt spring-neap cycle following the method of Latteux (Jeuken, 2000).

Figure 7 shows the development of the water level differences in macrocell 1 during this tidal cycle for the year 2002. The graph makes clear that both the primary and secondary

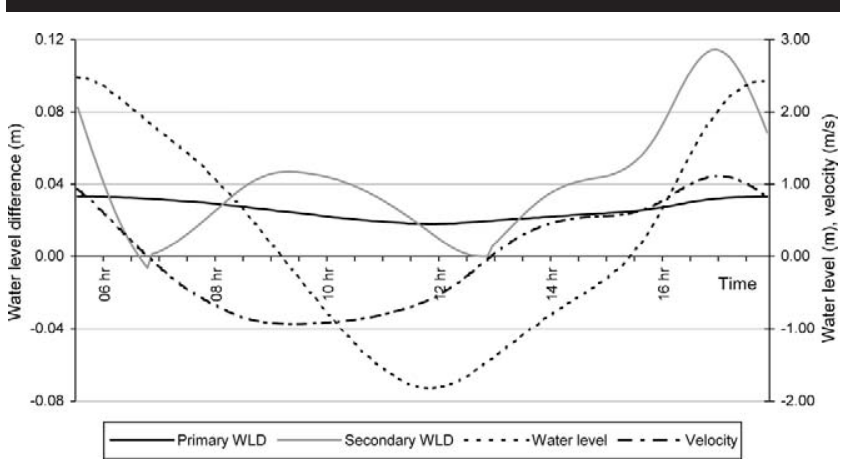

Figure 7. Typical development of water level, current velocity, and water level differences over a tidal cycle.

effects are most pronounced at or just after the moment of maximum flood flow. Because of the nonlinear dependency of sediment transport on current velocities, which are related by a power $n(\sim 2-5)$, it is assumed that the moments of maximum tidal flow are decisive in the formation of connecting channels. To be able to express the water level differences by a single yearly value, only the moment of maximum flood flow during the representative tide was considered in the analysis.

Next, the annual values of the connecting channel dimensions were plotted against the magnitude of the water level differences. The relationship between the two was expressed by the correlation coefficient $r$, which thus provides a measure of the relationship between main and connecting channels.

\section{Verification of Analytical Approach Against Numerical Model}

To gain confidence in the analytical assessment of the water level differences, the analytical approach was verified against a hydrodynamic simulation of the Western Scheldt, performed with the numerical model Delft3D-FLOW. The existing morphological model of the Western Scheldt was used for this purpose, the calibration and validation of which was extensively carried out in terms of water levels, discharges, and current velocities (Kuijper et al., 2004). The model was configured with tidal boundary conditions and bathymetry for the year 2002. Hence, the comparison between the analytical results and the Delft3D numerical model results was made for 2002 only.

Generally, a numerical model may have an uncertainty in the modelled water levels on the order of several percent. In the Western Scheldt model, this uncertainty falls in the same order of magnitude as the water level differences that are the focus of attention in this study. However, the main driving force for connecting channels is not the absolute tidal water levels but rather the local relative water level differences between two main channels. The physical processes responsible for these local differences (thus Coriolis effect, centrifugal effect, and difference in tidal wave propagation) are explicitly incorporated in Delft3D-FLOW (Stelling, 1983), which makes 
it possible to filter out the three mechanisms from the local absolute modelled water levels.

In the model, several observation points were specified, at which water levels were recorded during the representative tidal cycle. The Coriolis and centrifugal effects were derived from observation points on both sides of an individual main channel, where the effect of tidal wave propagation does not interfere with the local water level differences. The tidal wave propagation effect was derived from observation points on both ends of a straight section of a main channel, where the centrifugal effect does not interfere. In these runs, the Coriolis effect was switched off by locating the model at the equator.

For the same locations as the observation points in the model, the analytical water level differences were also determined, according to the approach described in the "Mechanisms Causing Water Level Differences" section. Figure 8 present the numerical against the analytical water level differences. This shows that the Coriolis effect is very accurately predicted, whereas the analytical centrifugal effect deviates a little more from the modelled results. The latter is especially the result of the difficulty in assessing the radius of curvature of the main channel exactly. For both mechanisms, a constant $1 \mathrm{~m} / \mathrm{s}$ cross-sectional averaged velocity was assumed in the analytical treatment. Although this assumption seems generally appropriate, at distinct locations in the estuary slightly higher cross-sectional velocities are observed. Extensive verification against the numerical model shows that both the Coriolis and centrifugal mechanisms are assessed with acceptable accuracy regardless of this assumption. Moreover, the expected range of variation in $\Delta h_{\text {long }}$ and $\Delta h_{\text {cross }}$ that would result from considering variable flow velocities over time and space would be of minor significance as compared to the accompanied variation in the wave propagation mechanism, both of which spring from changes in channel depth.

The relative water level differences due to tidal wave propagation between two main channels were not directly determinable from the numerical model because of local interference with other physical processes; however, the propagation times over a straight section of an individual main channel are accurately predicted (see Figure 8c). As the water level differences due to tidal wave propagation are a direct function of the wave propagation times over two main channels, it is assumed that $\Delta h_{w p}$ is likewise correctly assessed.

\section{RESULTS}

\section{Temporal Changes in Water Level Differences}

Figure 9 shows the temporal evolution of water level differences between the main channels for each macrocell at the moment of maximum flood. $\Delta h_{w p}$ is typically positive, indicating that the tidal wave propagation is fastest along the flood channels. This is the result of the shorter length of the flood channel as compared to the ebb channel, which outweighs the larger depth of the ebb channel in determining the propagation time along the channels. Only in macrocell 6 does the shallowness of the flood channel and large (artificial) depth of the ebb channel result in a negative $\Delta h_{w p}$. The
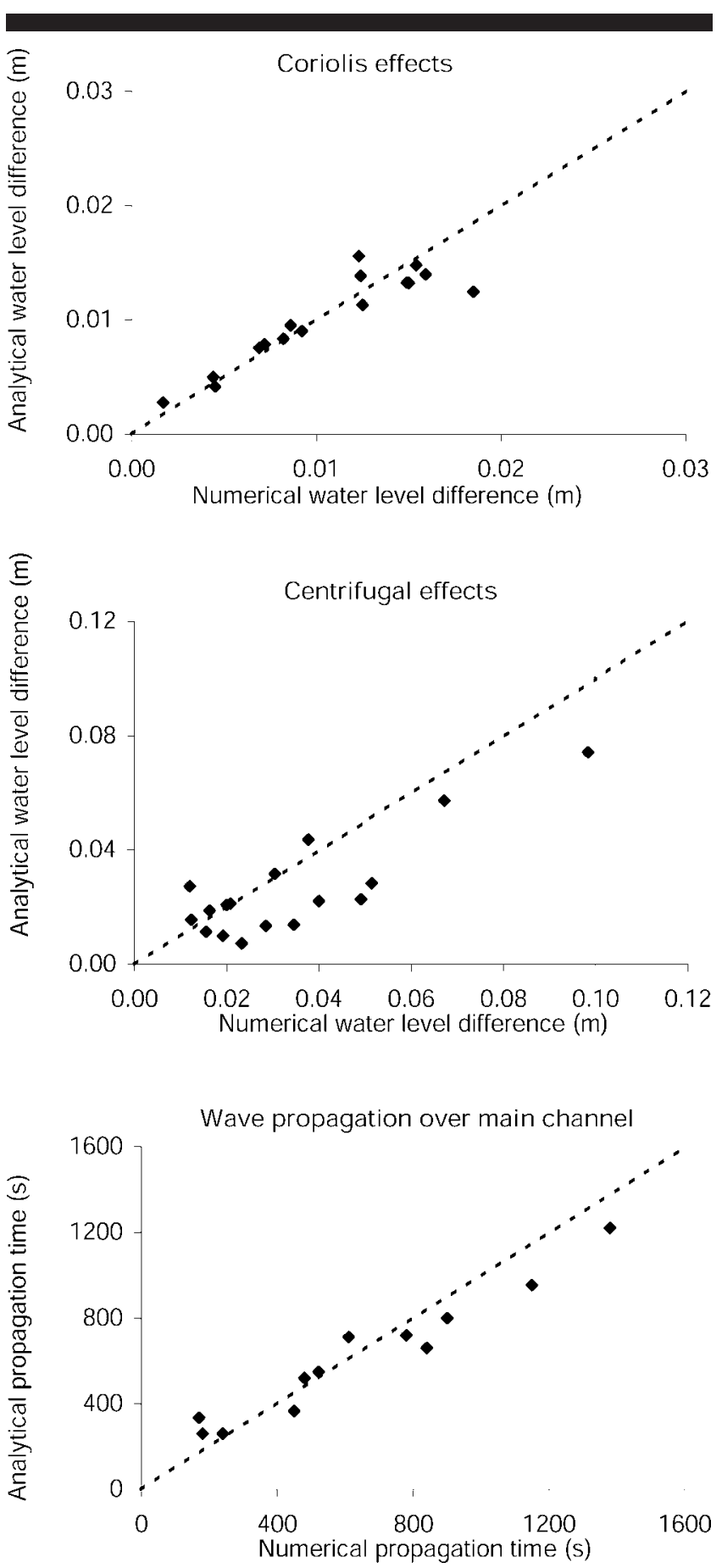

Figure 8. Comparison of numerical against analytical model results for (a) Coriolis effect, (b) centrifugal effects, and (c) wave propagation over a main channel.

magnitude of the longitudinal secondary effect, $\Delta h_{\text {long, }}$ at maximum flood is always positive, which implies that an additional head difference is enforced over the flood channel with respect to the ebb channel. With the exception of macrocell $4, \Delta h_{\text {long }}$ appears to be larger than the wave propagation effect. Conversely, the secondary transverse effect, 

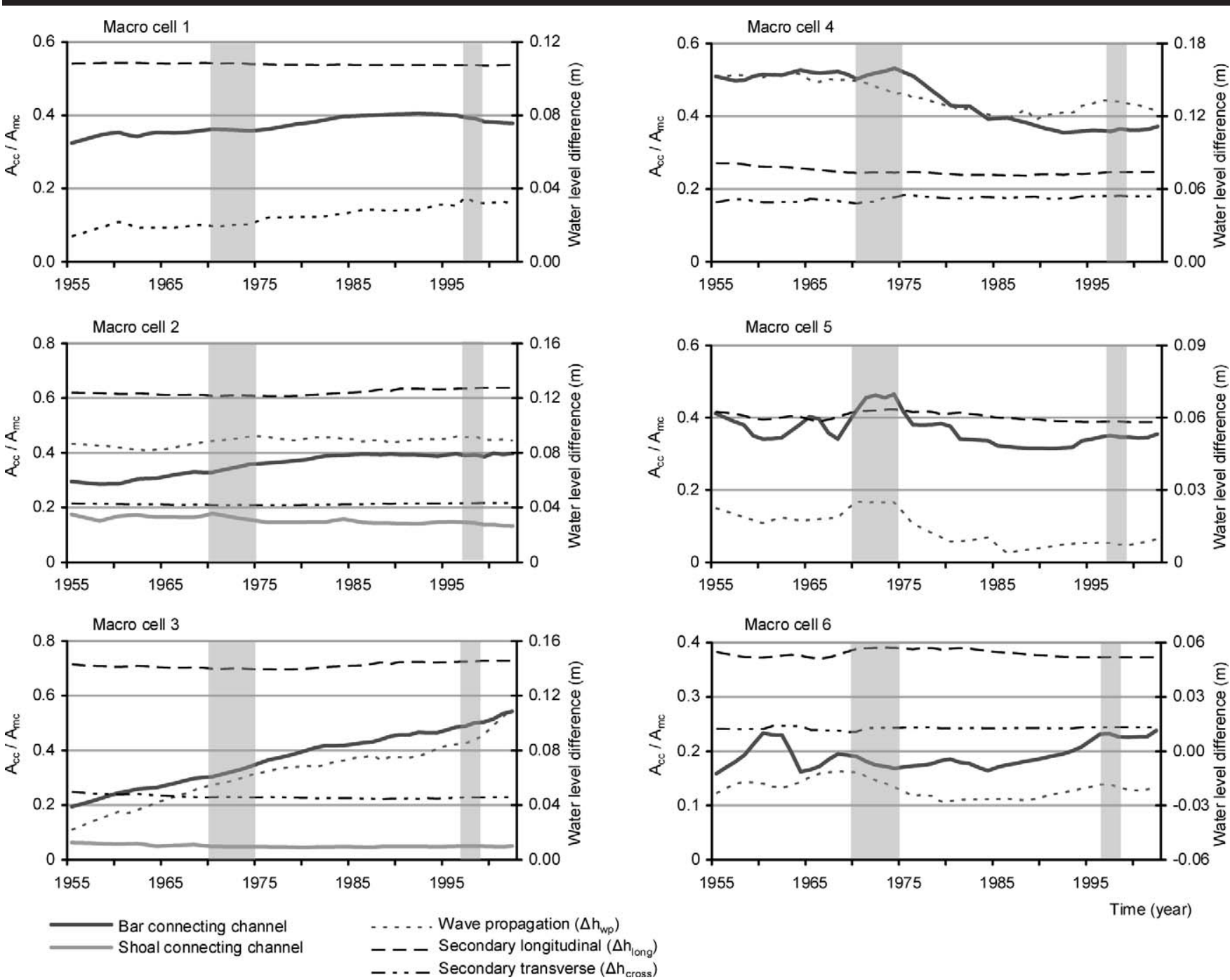

Figure 9. Development of water level differences and connecting channel size for each macrocell during the period 1955-2002. Water level differences were analytically assessed based on Equations 2, 5, and 6 at the moment of maximum flood during the annual representative tide. Cross-sectional areas of connecting channels were derived from the bathymetrical data set. Grey areas indicate the first (1970-1975) and second (1997-1998) deepening programme. Note that the y-axis scales differ.

$\Delta h_{\text {cross }}$, is of minor significance and is subordinate to both the primary and secondary longitudinal effect. In macrocells 1 and 5 , the transverse secondary effect does not exist, because of the absence of a shoal area between the main channels. In these macrocells, a single continuous transverse water slope is set up over both main channels.

$\Delta h_{w p}$ shows temporal variability on a time scale of years to decades, which is larger than the temporal variability of both $\Delta h_{\text {long }}$ and $\Delta h_{\text {cross. }}$ This is the result of the limited freedom of movement of the tidal channels; the construction of dikes along the estuary and channel reinforcements have essentially anchored their horizontal configuration. Thus, changes in the main channels reveal themselves mainly in channel depth, whereas the temporal variation in channel length, width, and radius is much less significant. The variability of $\Delta h_{w p}$ is hence largely the result of relative changes in $h_{a v}$ be- tween the main channels; the comparatively constant values of $\Delta h_{\text {long }}$ and $\Delta h_{\text {cross }}$ are the result of the nearly unchanging magnitude of $u, B$, and $R$. Obviously, $u$ was assumed constant in this study, whereas in practice it may vary over time as a response to changes in channel depth. Nevertheless, the anticipated temporal variation of $u$ would still have a minor impact on water level differences as compared to the temporal variation of $h_{a v}$, and hence of $\Delta h_{w p}$.

\section{Correlation Between Water Level Differences and Connecting Channels}

Because of the small temporal variation in $\Delta h_{\text {long }}$ and $\Delta h_{\text {cross }}$, it is expected that the variability in the dimensions of connecting channels can largely be associated with the variation in $\Delta h_{w p}$. This assumption is tested by correlating the 
Table 1. Correlation between water level differences and relative bar connecting channel size, expressed by $r$ values.

\begin{tabular}{|c|c|c|c|c|c|c|}
\hline \multirow[b]{2}{*}{ Correlation } & \multicolumn{6}{|c|}{ Macrocell } \\
\hline & 1 & 2 & 3 & 4 & 5 & 6 \\
\hline$\Delta \mathrm{h}_{w p}-A_{b c} / A_{m c}$ & 0.87 & 0.78 & 0.98 & 0.85 & 0.84 & 0.00 \\
\hline $95 \%$ confidence interval & $0.78-0.93$ & $0.64-0.87$ & $0.96-0.99$ & $0.74-0.91$ & $0.73-0.91$ & $-0.28-0.28$ \\
\hline$\Delta h_{\text {long }}-A_{b c} / A_{m c}$ & 0.81 & 0.54 & 0.63 & 0.58 & 0.72 & 0.65 \\
\hline $95 \%$ confidence interval & $0.68-0.89$ & $0.30-0.71$ & $0.42-0.78$ & $0.36-0.74$ & $0.55-0.83$ & $0.45-0.79$ \\
\hline$\Delta h_{\text {cross }}-A_{b c} / A_{m c}$ & $\mathrm{n} / \mathrm{a}$ & 0.28 & 0.81 & 0.63 & $\mathrm{n} / \mathrm{a}$ & 0.17 \\
\hline $95 \%$ confidence interval & $\mathrm{n} / \mathrm{a}$ & $0.00-0.52$ & $0.69-0.89$ & $0.42-0.78$ & $\mathrm{n} / \mathrm{a}$ & $-0.12-0.44$ \\
\hline$\Delta h_{\text {total }}-A_{b c} / A_{m c}$ & 0.86 & 0.87 & 0.98 & 0.82 & 0.87 & 0.10 \\
\hline $95 \%$ confidence interval & $0.76-0.92$ & $0.77-0.92$ & $0.97-0.99$ & $0.71-0.90$ & $0.77-0.92$ & $-0.19-0.37$ \\
\hline
\end{tabular}

Because of the absence of a shoal between the main channels, macrocells 1 and 5 do not exhibit a water level difference in transverse direction $\left(\Delta h_{\text {cross }}\right)$. $\Delta h_{\text {total }}=\Delta h_{w p}+\Delta h_{\text {long }}$.

annual analytically determined water level differences with the annual cross-sectional area of bar connecting channels $\left(A_{b c}\right)$. In the correlation analyses for macrocells 2 and 3, where shoal connecting channels as well as bar connecting channels exist, the shoal connecting channels $\left(A_{s c}\right)$ were treated separately.

It is probable that the absolute size of connecting channels adjusts in response to an expansion or to sedimentation of a macrocell as a whole, in addition to adjusting to variation in the depth ratio between the individual main channels. To eliminate this effect, $A_{b c}$ and $A_{s c}$ are normalized with respect to the cross-sectional area of the main channels, $A_{m c}\left(=A_{f c}\right.$ $\left.+A_{e c}\right)$, which is, similar to the connecting channels, referenced to $-2 \mathrm{~m}$ NAP. In the following correlation analyses, the head differences are treated as the independent parameter and are therefore plotted on the horizontal axis. The statistical significance of the relationship is expressed by the value of the correlation coefficient ( $\mathrm{r}$ ) and the associated $95 \%$ confidence intervals. Results of the correlation analyses for the individual macrocell are presented in Table 1 (bar connecting channels) and Table 2 (shoal connecting channels).

Focusing on the correlation between $\Delta h_{w p}$ and bar connectning channel dimensions, one notices that most macrocells show a fairly good fit to a straight trend line; $r$ values range between 0.78 and 0.98 , which points toward a relationship between the two quantities. Only macrocell 6 forms an exception to this rule with an $\mathrm{r}$ value of 0 . The relationship between both $\Delta h_{\text {long }}$ and $\Delta h_{\text {cross }}$ and connecting channel dimensions is less evident and in all cases smaller than $\Delta h_{w p}$. This supports the assumption that $\Delta h_{w p}$ is the main contributor to the temporal evolution of connecting channels. Figure $10 \mathrm{a}$ presents the resulting relationship between $\Delta h_{w p}$ and $A_{b c} / A_{m c}$ in the macrocells. It should be noted that macrocell 6 is not shown in this figure because of the absence of any correlation.
It appears that considering the wave propagation mechanism by itself does provide a satisfactory relationship between water level differences and bar connecting channels. However, this raises questions about the importance of the secondary mechanisms, in particular of $\Delta h_{\text {long }}$. The large water level differences due to this mechanism often exceed $\Delta h_{w p}$ in magnitude, and it is therefore very plausible that these also play a role in maintaining the bar connecting channels. Indeed, the numerical modeling exercise showed that the secondary mechanisms may have a decisive role in determining the direction of the water level differences between two main channels and consequently cannot be ignored. Both $\Delta h_{\text {long }}$ and $\Delta h_{w p}$ act in a longitudinal direction over the bar area in the flood channel and can therefore be added to arrive at a total longitudinal head $\left(\Delta h_{\text {total }}\right) . \Delta h_{\text {cross }}$ is ignored in this total head difference, as it acts in a transverse direction and not over the bar area, where bar connecting channels occur. $\Delta h_{\text {total }}$ is thus obtained by:

$$
\Delta h_{\text {total }}=\Delta h_{w p}+\Delta h_{\text {long }} .
$$

Correlating $\Delta h_{\text {total }}$ and relative $A_{b c}$ results in a similar good coherence as found when considering $\Delta h_{w p}$ only (Table 1). Again, no correlation is found in macrocell 6; for the remaining macrocells $\mathrm{r}$ ranges between 0.82 and 0.98 . Figure $10 \mathrm{~b}$ presents the relation between bar connecting channel dimension and $\Delta h_{\text {total }}$ for the macrocells.

The correlation between water level differences and shoal connecting channels (Table 2) does not show the same clear trend as for the bar connecting channels. It was assumed that $\Delta h_{\text {cross }}$ would play a larger role in maintaining shoal channels, because of the transverse direction of $\Delta h_{\text {cross }}$ acting over the shoal between two main channels. However, in macrocell 2, the strongest correlation is found with $\Delta h_{\text {total }}$ with an $\mathrm{r}$ value of -0.88 . This negative relationship implies that with in-

Table 2. Correlation between water level differences and relative shoal connecting channel size, expressed by $r$ values.

\begin{tabular}{|c|c|c|c|c|}
\hline \multirow[b]{2}{*}{ Correlation } & \multicolumn{2}{|c|}{ Macrocell 2} & \multicolumn{2}{|c|}{ Macrocell 3} \\
\hline & $\mathrm{r}$ & 95\% Confidence Interval & $\mathrm{r}$ & 95\% Confidence Interval \\
\hline$\Delta h_{w p}-A_{s c} / A_{m c}$ & -0.60 & $-0.76--0.38$ & -0.75 & $-0.85--0.59$ \\
\hline$\Delta h_{\text {long }}-A_{s c} / A_{m c}$ & -0.83 & $-0.90--0.72$ & 0.00 & $-0.28-0.28$ \\
\hline$\Delta h_{\text {cross }}-A_{s c} / A_{m c}$ & -0.69 & $-0.82--0.51$ & 0.90 & $0.83-0.94$ \\
\hline$\Delta h_{t o t}-A_{s c} / A_{m c}$ & -0.88 & $-0.93--0.80$ & -0.71 & $-0.83--0.53$ \\
\hline
\end{tabular}

Because of the absence of a shoal channel between the main channels in macrocells $1,4,5$, and 6 , they are not included in the table. 

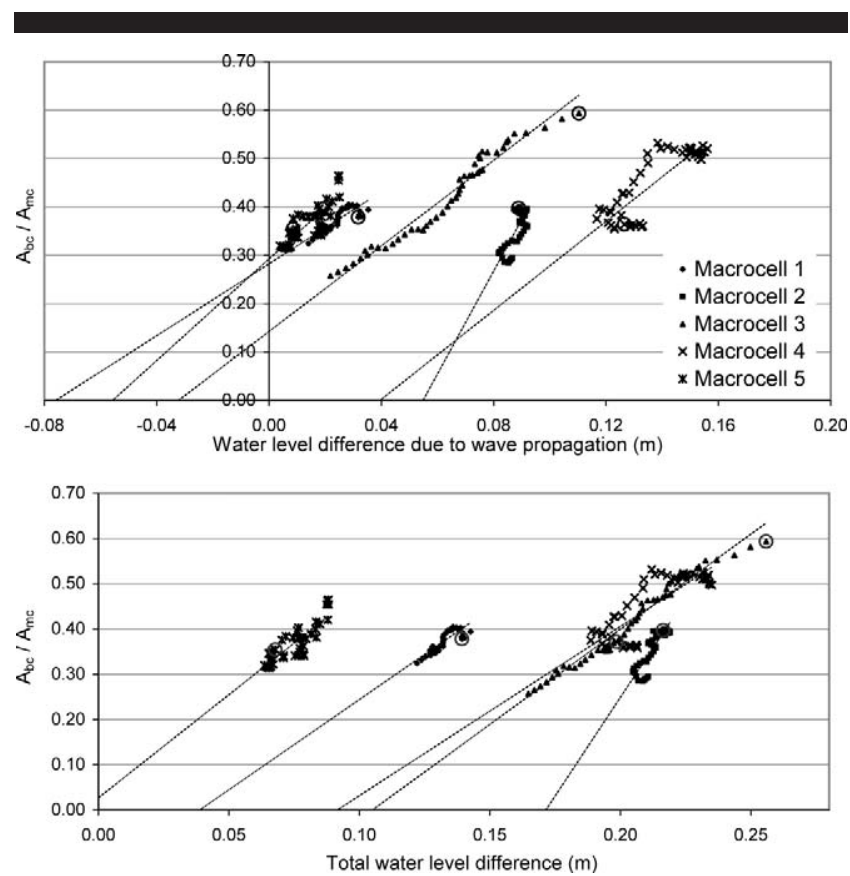

Figure 10. Relation between (a) water level differences due to wave propagation $\left(\Delta h_{w p}\right)$ and relative size of bar connecting channels, and (b) total water level differences $\left(\Delta h_{\text {total }}\right)$ and relative size of bar connecting channels. Circled values mark the position in 2002. The crossing of the best-fit line with the $\mathrm{x}$-axis is an indication of the critical water level difference for each macrocell. Note that macrocell 6 is not shown because of the absence of any correlation.

creasing $\Delta h_{\text {total }}$ the size of the shoal channel decreases. This is presumably related to a negative feedback mechanism that exists between bar connecting channels and shoal connecting channels in this macrocell, which will be further discussed below. Although the assumed higher influence of $\Delta h_{\text {cross }}$ does not apply in macrocell 2 , in macrocell 3 it does seem to relate well to the shoal channels with an $r$ value of 0.90 . As only two shoal connecting channels existed in the estuary in the analysis period that seem to have distinct forcing and feedback mechanisms, the focus of the remainder of this article lies on the bar connecting channels.

\section{Evolution of Connecting Channels and Impact of Dredging Operations}

It is evident from Figure 9 that human interference has had more impact on the development of $\Delta h_{w p}$ than on $\Delta h_{\text {long }}$ and $\Delta h_{\text {cross. }}$ Both secondary mechanisms appear to be unaffected by the large-scale dredging operations (indicated by the grey areas). On the other hand, the effects on $\Delta h_{w p}$ can be noted during and after the two deepening programmes in several macrocells, the clearest examples being macrocells 4 and 5. Below, the evolution of $\Delta h_{w p}$ and connecting channel dimensions is discussed for each macrocell to highlight the impact of natural and human alterations of the main channels on the development of the connecting channels.

\section{Macrocell 1}

Macrocell 1 shows a steady increase in $\Delta h_{w p}$ and a similar increasing trend in bar connecting channels. The growth in head differences has resulted in the expansion of the connecting channel area, and over the years the bar area in the flood channel has deepened and evolved from an intertidal into a subtidal bar. The increase in connecting channel size between the mid-1960s and mid-1980s was moderated by regular sediment dumpings in the connecting channels after the first deepening programme. During the early 1990s, the cross-sectional area of the connecting channels was at its maximum, after which it started to decline. This can possibly be related to the degeneration of a large connecting channel that had existed in the bar area since 1964, which started to show sedimentation around 1984. The natural sedimentation process accelerated during the second deepening programme, even though the head difference continued to grow in this period. The accelerated degeneration is probably the result of extensive sediment dumping in the channel during and after the second deepening. Currently, the relative size of the bar connecting channels in the macrocell is larger than in 1955, and the bar area has thus eroded significantly in that period.

\section{Macrocell 2}

Macrocell 2 exhibits very dynamic bar connecting channels that show the largest migration rates in the Western Scheldt. Moreover, it is the only remaining macrocell in the estuary with an active migrating shoal connecting channel crossing the intertidal area between the main channels. Jeuken (2000) already recognized interrelated behaviour between the shoal connecting channel and the bar connecting channels in the macrocell; the two channel types show an opposite morphologic evolution caused by a negative feedback mechanism. She noticed that this behaviour is manifest at a timescale of years to a few decades. The bar connecting channels correlate fairly well with $\Delta h_{t o t}(\mathrm{r}=0.87)$, whereas there exists a negative relation between the shoal channels and $\Delta h_{t o t}(\mathrm{r}=$ $-0.88)$. Although the former correlation may well be physical, the latter correlation might be more the result of the large interdependence between the two channel types than directly from the effect of changes in $\Delta h_{t o t}$.

The long-term development of the main channels in macrocell 2 is characterized by natural erosion of the flood channel, even though sediment is being dumped here, and net erosion of the ebb channel, where sediment is being dredged. Overall, the main channels have only experienced small morphological changes relative to each other: this is reflected in the small variation in $\Delta h_{w p}$ over time. Nevertheless, the cross-sectional area of the bar channels shows a steady increase between 1960 and 1985 . The evolution of the bar channels may partly be related to the natural sedimentation of the shoal channel that occurred in that period (Jeuken, 2000), which could explain the observed "swing" around the main trend line shown in Figure 10 and the rather poor correlation in the macrocell. 


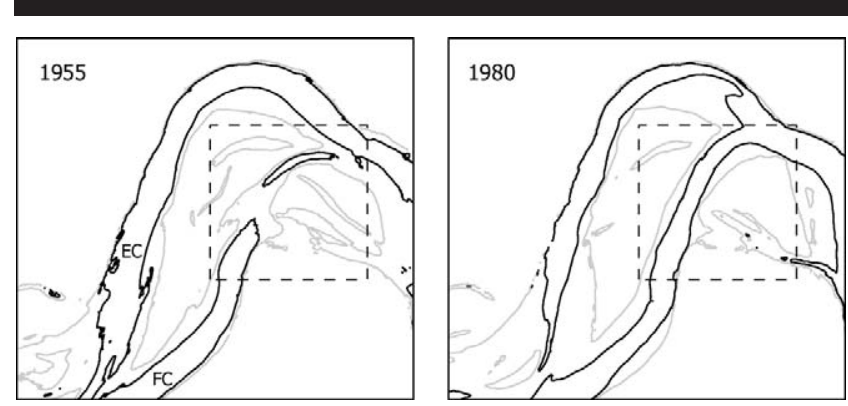

Figure 11. Macrocell 3: expansion of a bar connecting channel. The channel breaches through the bar area and forms a full connection between the main flood channels in macrocell 3 and 4 in 1980. Dark line = $-14 \mathrm{~m}$ contour line, light line $=-2 \mathrm{~m}$ contour line, $\mathrm{EC}=$ ebb channel, $\mathrm{FC}=$ flood channel

\section{Macrocell 3}

Macrocell 3 provides the clearest sign of the existing relationship between main channel evolution and connecting channels; there is a strong linear coherence between $\Delta h_{w p}$ and relative $A_{b c}(\mathrm{r}=0.98)$. Due to the (natural) erosion of the flood channel and simultaneous sedimentation of the ebb channel, $\Delta h_{w p}$ steadily increased between 1955 and 2002, which was accompanied by a large expansion of a bar connecting channel. This process eventually led to a natural bend cut-off of the main ebb channel (Figure 11) and to the flood channel gradually becoming more dominant. As a result, the main navigation channel has been diverted from the ebb channel through the flood channel since 1980. Just after completion of the second deepening programme, the inclining trend of $\Delta h_{w p}$ was further accelerated by an increased dumping load in the ebb channel and associated erosion of the flood channel.

It should be mentioned that the expansion of the bar connecting channel may have reinforced the evolution process of the main channels by enhancing the erosion of the flood channel because of its increased discharge capacity. As a consequence, the cause-effect relation is not entirely one-directional in this macrocell. However, the initiation of the morphological changes in the macrocell was the result of the natural erosion/sedimentation of the main channels, which was only later followed by the reinforcing feedback mechanisms of the connecting channel.

During the analysis period, the shoal connecting channel has slowly reduced in size. It is believed that before the start of this analysis period, when the main ebb channel was still dominant, the macrocell featured active migrating shoal channels. However, since the change in the system to a predominant flood channel, the shoal connecting channel has presumably become redundant from its function of redistributing the ebb tidal flow to the main ebb channel and has slowly been degenerating since. As a result, the channel may have become less responsive to the original forcing mechanisms. Regardless, there is a correlation between $\Delta h_{\text {cross }}$ and the shoal channel of $r=0.90$. This is related to a coinciding decreasing trend in $\Delta h_{\text {cross }}$ because of changes in main channel width, which is not thought to be the cause of the decrease in shoal channel size.

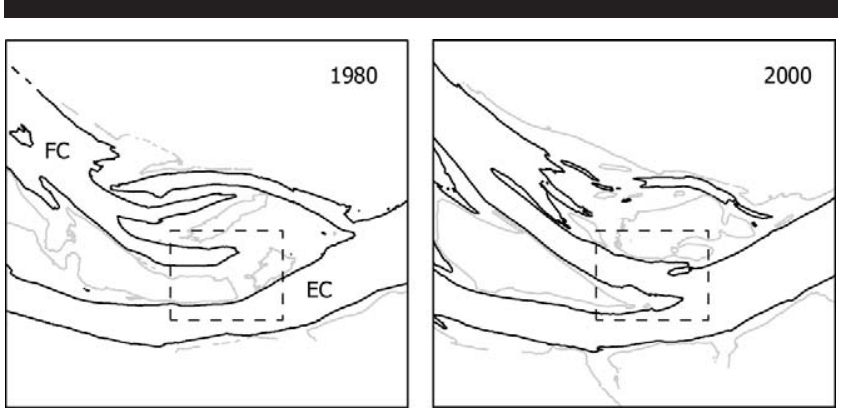

Figure 12. Macrocell 4: absence of a full connection between the main ebb and flood channel in 1980. In 2000, a newly formed small bar connecting channel reconnects the main channels. Dark line $=-4 \mathrm{~m}$ contour line, light line $=-2 \mathrm{~m}$ contour line, $\mathrm{EC}=$ ebb channel, $\mathrm{FC}=$ flood channel.

\section{Macrocell 4}

In macrocell $4, \Delta h_{w p}$ has been declining since the start of the analysis period, which is related to a deepening of the main ebb channel. This is understood to be a natural process that already commenced in 1900 (Jeuken, 2000) and has been accelerated by the deepening and maintenance dredging of the ebb channel bar since the early 1970s. The reduction in $\Delta h_{w p}$ was followed by a decrease in connecting channel size. Figure 9 demonstrates that the main decrease in the cross section of bar connecting channels started after completion of the first deepening programme, and it is believed that this was an adjustment to the new conditions after the dredging operation. The steep decline in size between 1975 and 1985 almost led to a disconnection of the main ebb and flood channel; around the early 1980s, there was no longer a fully open connection between the main channels below the low tidal limit (Figure 12). The natural erosion of the flood channel and accompanying increase in $\Delta h_{w p}$ between 1988 and 1995 stopped this process and resulted in a new breach through the bar area around 1990. In 1997, the start of the second deepening period, the trend in $\Delta h_{w p}$ reversed again as a result of the deepening of the sills in the ebb channel and has been decreasing since.

\section{Macrocell 5}

Macrocell 5 shows large fluctuations in $\Delta h_{w p}$ over time, related to intensive dredging and dumping activities in its channels. In the decade after the first deepening, a large reduction in $\Delta h_{w p}$ is visible, possibly related to the adaptation of the main channels to the deepening of the sills in the ebb channel. This was accompanied by a reduction of the relative size of bar connecting channels. Since 1994, a small increase in relative $A_{b c}$ can be observed again. This follows an increase in $\Delta h_{w p}$ that started in 1986, related to the natural erosion of the flood channel. This process was accelerated after the second deepening when sediment dumping in the flood channel was halted.

\section{Macrocell 6}

In macrocell $6 \Delta h_{w p}$ is negative, which implies that the tidal wave propagation is fastest along the ebb channel. This is the 
result of the shallowness of the flood channel and the large (artificial) depth of the ebb channel. The sills in the ebb channel have been dredged since as early as 1922 and were further lowered during both deepening programmes. In the years after these operations, the ebb channel showed a large expansion. At the same time, sediment was dumped in the flood channel, which hence experienced sedimentation. Naturally, however, the flood channel has a tendency to erode, which explains the gradual increase in $\Delta h_{w p}$ between 1955 and 1970 and between 1983 and 2002 .

Before 1955, macrocell 6 exhibited both shoal connecting channels and bar connecting channels. Nowadays, no shoal channels occur, whereas the formation of bar channels is obstructed by the presence of a training wall that guides the ebb channel. Relative to the main channels, the cross section of the connecting channels remains fairly stable during the analysis period, whereas $\Delta h_{w p}$ shows significant variation. The correlation between the two variables is therefore very poor: macrocell 6 forms the only example where no correlation between $\Delta h_{w p}$ and connecting channel dimensions can be found $(\mathrm{r}=0.0)$. The absence of a relationship can possibly be explained by the presence of the training wall. However, leaving out all data after 1968, the year of the construction of the wall, does not result in a much better fit ( $\mathrm{r}$ increases to 0.26 ); the trend remains unconfirmed.

\section{DISCUSSION}

The correlation between connecting channels and head differences shows that connecting channels are, as hypothesized, sustained by water level differences between the main channels. In principle, this is an obvious relationship: water level differences force water motion-hence sediment transport-that creates the connecting channels. The relationship therefore describes the state of the system as a function of its forcing. However, it also shows that other factors must play a role in defining the size of connecting channels: the results for the individual macrocells are not intercomparable, and it is probable that factors such as macrocell length, width, or grain size have to be taken into account for this. Furthermore, the interaction between shoal channels and bar channels influences connecting channel size, an aspect that was not explored in-depth in this study.

As the magnitude of the longitudinal component of the secondary mechanisms $\left(\Delta h_{\text {long }}\right)$ is in many cases larger than the primary mechanism $\left(\Delta h_{w p}\right)$, the study hints at the importance of the secondary mechanisms toward sustaining connecting channels. The secondary mechanisms are relatively constant with time, as they are to a large extent related to the macroscale (fixed) geometry of the estuary. Therefore, the temporal variation in the total head differences is primarily the result of variation in the primary mechanism, which, in turn, is largely determined by changes in the main channel depth.

\section{Human Influence on the Presence of Connecting Channels}

The above statement implies that by changing the depth ratio between an ebb and flood channel pair by dredging and dumping activities, mankind may have a large impact on the evolution of connecting channels. This can indeed be observed in some macrocells, where the connecting channel size seemed to respond to the altered hydrodynamic conditions during and after the large-scale dredging operations. Especially in the eastern macrocells $(4,5$, and 6$)$, the equilibrium between the main channels was disturbed by dredging of the sills in the ebb channel and sediment disposal in the flood channel. This led to an overall declining trend in $\Delta h_{w p}$, which in macrocells 5 and 6 is opposite to the expected natural trend; Jeuken et al. (2004) showed that the flood channels in these macrocells have a natural tendency to erode and the ebb channels to fill in, but human operations have reversed this process. The decline in $\Delta h_{w p}$ during the analysis period was thus largely the result of human interventions. In macrocell 6 , this effect is overshadowed by the interference of the training wall, which strongly influenced the natural evolution of the connecting channels.

\section{Limits on the Presence of Connecting Channels}

Connecting channels are considered to play a vital role in a multichannel system by stimulating the ecology and navigability of the estuary. As a consequence, their preservation is an important issue in the management of the Western Scheldt. Before being able to incorporate connecting channels in a management strategy, the boundary conditions for their presence need to be identified. Focussing on bar connecting channels, their existence is limited between two extremes:

- The upper limit on the presence of bar connecting channels can be visualized as an extreme expansion of a bar connecting channel such that it becomes part of the main flood channel. This happens in case $\Delta h$ becomes excessively large. The flood channel would then form a shortcut through the inner bend of the ebb channel and might eventually become dominant, similar to what happened in macrocell 3 (Figure 11). This upper limit can only be reached when the flood channel erodes significantly and/or the ebb channel experiences extensive sedimentation. Apart from macrocell 3 , this process cannot be observed anywhere in the estuary at present, and with the continual dredging of the ebb channel, it is unlikely that the upper limit on the presence of bar connecting channels will be reached in any macrocell in the near future.

- The lower limit on the presence of connecting channels can be visualized as a complete disappearance of a connecting channel due to sediment deposition in the channel. This happens when $\Delta h$ becomes excessively small as a result of an increasing shallowness of the flood channel and/or deepening of the ebb channel. With the present dredging and dumping activities in the estuary, such a declining tendency is likely to be encouraged in coming years. Any decline in $\Delta h$ is accompanied by a move toward the lower limit on the presence of connecting channels. Between 1955 and 2002 , the development of connecting channels already approached this limit on a few occasions; in macrocell 4 , a full connection between the main channels was lost around 1980 (Figure 12), the initial sign of degeneration of connecting channels. Also, connecting channels are nearly ab- 
Table 3. Critical and current water level difference due to wave propagation in 2002.

\begin{tabular}{crcc}
\hline \hline Macrocell & $\Delta h_{\text {crit }}(\mathrm{m})$ & $\Delta h_{w p}(\mathrm{~m})$ & $\Delta h_{w p}-\Delta h_{\text {crit }}(\mathrm{m})$ \\
\hline 1 & -0.08 & 0.03 & 0.11 \\
2 & 0.06 & 0.09 & 0.03 \\
3 & -0.03 & 0.11 & 0.14 \\
4 & 0.04 & 0.13 & 0.09 \\
5 & -0.06 & 0.01 & 0.07 \\
\hline
\end{tabular}

sent in macrocell 6 at present, whereas previously they existed here, too.

As the field data only provide a limited number of examples on the lower limits on connecting channels, the theoretical limits are explored here instead. These can be found by extrapolating the best-fit line for each data set, shown in Figure 10. The crossing of this line with the $\mathrm{x}$-axis indicates the theoretical critical head difference $\left(\Delta h_{\text {crit }}\right)$ that would result in an absence of connecting channels. Figure 10a is the most appropriate for investigating these limits in relation to estuarine management, because dredging and dumping activities mainly manipulate $\Delta h_{w p}$. The critical head difference obtained from this graph and the magnitude of $\Delta h_{w p}$ in 2002 for each macrocell are given in Table 3 . It should be noted that the critical values presented in this table should be regarded as indicative values only. However, the difference between the two values can be compared with anticipated values of $\Delta h_{w p}$ after proposed future dredging operations to give a rough indication of the susceptibility of the macrocell toward degeneration of its connecting channels.

Macrocell 2 appears to be most vulnerable as the difference between $\Delta h_{\text {crit }}$ and $\Delta h_{\text {total }}$ is smallest. However, the macrocell has experienced limited temporal variability of $\Delta h_{w p}$ in the past, which is unlikely to change significantly in the coming years as a result of dredging operations in the main ebb channel. To reach the critical water level difference, the full ebb channel would have to be deepened by $1 \mathrm{~m}$ and the flood channel would have to reduce in depth by a similar amount. Such changes are larger than the historical variation in main channel depth over the past 50 years and will probably not be instigated by the future deepening programme. Similarly, in macrocells 1,3 , and 5, the main ebb and flood channels would have to erode or shoal more than they have in the past half century to reach the critical value.

In macrocell 4 , on the other hand, there appears to be an imminent threat to the connecting channels. Here, the main ebb and flood channels already tended to lose a full connection around the mid 1980s. As a result of erosion of the flood channel, a new breach was formed and the connection reestablished, but it was demonstrated that degeneration of connecting channels in this macrocell is not unlikely. An increase in depth of the ebb channel by $2 \mathrm{~m}$ and a simultaneous reduction of the flood channel by $1.5 \mathrm{~m}$ would reduce $\Delta h_{w p}$ enough to reach the critical head difference. Such alterations to the main channels are not improbable regarding the large adaptation of the channels in the 15 years after the first deepening programme $(2.5 \mathrm{~m}$ and $1 \mathrm{~m}$, respectively).

From the above observations, one may conclude that, with the exception of macrocell 4 , there is no immediate threat for connecting channels to disappear in the estuary. However, the discussion is based on the theoretical water level difference that will lead to a complete absence of connecting channels. In practice, the connecting channels would already have silted up considerably, exhibited a reduction in dynamic migratory behaviour, and lost their full (subtidal) connection between the main channels long before reaching this critical state. Bearing in mind the aspiration to preserve the multichannel system and its dynamic character, both the loss of an open connection between the main channels and reduction of the migration rates of connecting channels are undesirable. The theoretical critical limit is therefore beyond the desirable minimum state of the connecting channels from management perspective, and an adequate definition of the lower tolerable limit is thus to be defined.

\section{Incorporation into Management Strategy}

An important starting point in the Western Scheldt management is understanding the action-reaction between human interventions (here, mining/dredging/dumping) and their impacts (here, presence of connecting channel). By having established the relation between main channel development and connecting channel development, it can be said with certain confidence that some of the past observed changes in the connecting channels were linked to human interventions.

It is now proposed to adopt a monitoring programme for connecting channels; by regularly assessing their presence and size, they may provide important information on the changes in the dynamic equilibrium of the multichannel system and thus give a signal of the state of the estuary. At the same time, it is proposed to monitor the water level difference between an ebb channel and flood channel pair by following a similar approach as described in this article. This will provide signs of changes in the forcing mechanism of the connecting channels and may thus predict future effects in an early stage. To limit further undesired impacts, this knowledge can be used to assess future dredge and dumping actions, to reconsider the dredge spoil dump locations, or to plan alternative dumping strategies.

This approach implies that the desired state of the Western Scheldt is one with the presence of connecting channels. However, the desired minimum size, type, and behaviour of the connecting channels has not been specified yet because of a lack of knowledge. These points are still open for further research.

\section{Recommendations for Improvements and Further Research}

In the present study, the six macrocells in the Western Scheldt were treated separately and a unique relationship for each of them was established. In the search for a general relationship applicable to all macrocells, it appeared that more factors must be considered than those that were included in this simplified geometrical analysis. It would be worth investigating the role of these factors-e.g., grain size, estuary width, head differences over a tidal cycle, and accurate 
current velocity data-in determining the size of connecting channels. In this respect, it would also be informative to investigate the presence of connecting channels in other estuaries with a multichannel system (e.g., The Humber Estuary, U.K.) and to compare these systems with the specific results found for the Western Scheldt system.

Another aspect that would be worth addressing is the presence and the role of shoal connecting channels. As shoal connecting channels in particular have disappeared in the estuary in the past century, it would be useful to better understand the relationship between shoal connecting channels and water level differences. Moreover, the interrelated behaviour between shoal connecting channels and bar connecting channels would need further investigation: the mutual buffering mechanism that they form makes the connecting channels respond differently to hydrodynamic changes in a macrocell with shoal connecting channels than in a macrocell without shoal connecting channels.

Finally, it was noted that there appears to be a delay in the morphological adjustment of the connecting channels to changes in water level differences. In some cases, an improved relationship between connecting channels and water level differences was found by taking this morphological inertia into account. It seems to be an important factor, especially in macrocell 4: $\mathrm{r}$ improves from 0.82 to 0.96 by assuming a response time lag of 6 years. In other macrocells, introducing such a time lag has a similar but less strong influence on the correlation results. This time lag falls within the temporal scale over which the characteristic morphological behaviour of connecting channels is manifest, which is on the order of 1-10 years (Jeuken, 2000), and would imply that changes in head differences are indeed the driving mechanism for, and not the result of, changes in connecting channels. However, the cause-effect relation is not always so clear cut, and in some cases connecting channels are thought to have had a controlling effect on main channel evolution too, such as in macrocell 3. The time lag effect and cause-effect relation have not been explored in great depth and would be worth more attention.

\section{CONGLUSIONS}

In the Western Scheldt, connecting channels originate from water level differences between two main channels. Such differences are caused by and are the sum of (1) differences in tidal wave propagation in the main channels, (2) centrifugal forces, and (3) Coriolis forces. The magnitude of these mechanisms was determined by considering characteristic geometrical properties of the main channels. This enabled a relationship to be identified between water level differences and connecting channel cross-sectional area. This relationship helps to explain the evolution of connecting channels on the basis of morphological changes of the main channels.

The key parameter that plays a role in temporal variation in water level differences is the difference in depth between a main ebb and flood channel. This implies that dredging and dumping operations may affect the evolution of connecting channels by bringing about changes in the depth ratio of the main channels.
A general downward trend in connecting channel size and dynamics has been observed in the Western Scheldt over the past century, which—at least to some extent—can be attributed to the intensive human interference in the channel system. A third deepening programme has been proposed for 2008 that will lower the bar areas in the navigation channel by an additional $1.5 \mathrm{~m}$. This is expected to induce a further tilting of the channel system towards the ebb channel, which is likely to be accompanied by a decrease in the size and/or number of connecting channels in parts of the Western Scheldt. Macrocell 4 appears to be most vulnerable; there is a considerable threat that connecting channels will disappear in this macrocell. In designing future dredging operations, a better evaluation of the impacts on connecting channels is possible given the insights that were gained in this study.

\section{LITERATURE CITED}

Jansen, P. Ph., 1994. Principles of River Engineering: The Non-Tidal Alluvial River. Delft, The Netherlands: Delft University Press, $509 p$.

Jeuken, M.C.J.L., 2000. On the Morphologic Behaviour of Tidal Channels in the Westerschelde Estuary. Utrecht, The Netherlands: University of Utrecht, Ph.D. thesis, 378p.

Jeuken, M.C.J.L. and Wang, Z.B., 2009. Impact of dredging and dumping on the stability of ebb-flood channel systems-part 2: observations. Submitted to the Journal of Coastal Engineering.

Jeuken, M.C.J.L.; Wang, Z.B.; Van der Kaaij, T.; Van Helvert, M.; Van Ormondt, M.; Bruinsma, R., and Tánczos, I., 2004. Morfologische Ontwikkeling in het Schelde Estuarium bij Voortzetting van het Huidige Beleid en Effecten van een Verder Verdieping van de Vaargeul en Uitpoldering langs de Westerschelde. Consortium Arcadis-Technum [In Dutch]. Delft, The Netherlands: WL | Delft Hydraulics, 211p.

Kuijper, C.; Steijn, R.C.; Roelvink, J.A.; Van der Kaaij, T., and Olijslagers, P., 2004. Morphological Modelling of the Western Scheldt. Delft, The Netherlands: WL | Delft Hydraulics.

Stelling, G.S., 1983. On the Construction of Computational Methods for Shallow Water Flow Problems. Delft, The Netherlands: Technical University of Delft, Ph.D. thesis, 227p.

Van den Berg, J.H.; Jeuken, M.C.J.L., and Van der Spek, A.J.F., 1996. Hydraulic processes affecting the morphology and evolution of the Westerschelde Estuary. In: Nordstrom, K.F. and Roman, C.T. (eds.), Estuarine Shores: Evolution, Environment and Human Alterations. London: John Wiley \& Sons Ltd, pp. 157-184.

Van Koningsveld, M.; Davidson, M.A., and Huntley, D.A., 2005. Matching science with coastal management needs: the search for appropriate coastal state indicators. Journal of Coastal Research, 21(3), pp. 399-411.

Van Rijn, L., 1990. Principles of Fluid Flow and Surface Waves in Rivers, Estuaries, Seas and Oceans. Amsterdam, The Netherlands: Aqua Publications.

Van Veen, J. 1950. Ebb and flood channel systems in the Netherlands tidal waters. Journal of the Royal Dutch Geographical Society, 67, pp 303-325.

Wang, Z.B.; Jeuken, M.C.J.L.; Gerritsen, H.; De Vriend, H.J., and Kornman, B.A., 2002. Morphology and asymmetry of the vertical tide in the Western Scheldt Estuary. Continental Shelf Research, 22 , pp. 2599-2609.

Wang, Z.B.; Jeuken, M.C.J.L., and Winterwerp, J.C., 2009. Impact of dredging and dumping on the stability of ebb-flood channel systems-part 1: theory. Submitted to the Journal of Coastal Engineering.

Winterwerp, J.C.; Wang, Z.B.; Stive, M.J.F.; Arends, A.; Jeuken, C.; Kuijper, C., and Thoolen, P.M.C., 2001. A new morphological schematization of the Western Scheldt Estuary, The Netherlands. In: Proceedings of the 2nd IAHR Symposium on River, Coastal and Estuarine Morphodynamics (Obihiro, Japan), pp. 525-534. 